\title{
Multi-step microfludic reactor for the synthesis of hybrid nanoparticles
}

\author{
Malik Abdul Wahab ${ }^{1}$ and E Yegan Erdem ${ }^{1,2}$ (D) \\ ${ }^{1}$ Bilkent University, Mechanical Engineering Department, Ankara, Turkey \\ ${ }^{2}$ National Nanotechnology Research Center, Ankara, Turkey \\ E-mail: yeganerdem@bilkent.edu.tr
}

Received 10 March 2020, revised 21 April 2020

Accepted for publication 28 April 2020

Published 8 June 2020

\begin{abstract}
We introduce a multi-step microfluidic reactor for the synthesis of hybrid nanoparticles. As part of this study, nanoparticles composed of chitosan and iron-oxide are synthesized at room temperature by forming sequential droplets of reagents in the microreactor followed by merging and mixing them in a step by step process. The obtained nanoparticles were characterized by transmission electron microscopy, Fourier transform infrared spectrometry, and energy dispersive X-ray analysis. Results were compared with nanoparticles of the same composition synthesized with batch-wise conventional techniques. As a result, the obtained nanoparticles showed better size distribution. This microfluidic device can be used for the synthesis of other types of nanoparticles that require multi-step procedures.
\end{abstract}

Keywords: microfluidics, microreactor, droplet-based flow, droplet merging, nanoparticle synthesis, hybrid nanoparticles

(Some figures may appear in colour only in the online journal)

\section{Introduction}

Hybrid nanomaterials are composed of two or more types of materials that show multifunctional behavior which makes them promising for applications in branches of science, engineering and medicine [1-3]. It is very crucial to synthesize these materials monodispersely as their properties are highly dependent on their size, shape and composition. Mostly nanomaterials are synthesized with conventional batch-wise methods, however these techniques have limitations in maintaining uniform reaction conditions such as temperature, concentration, residence time and therefore they usually result in polydisperse size distributions. On the other hand microfluidic reactors are shown to provide precise control over these parameters resulting in much monodisperse products [4-6] with reduced sample usage and minimized exposure to toxic materials [7].

Synthesis of nanoparticles using microfluidic systems has been studied earlier and there are also studies that focused on the synthesis of hybrid nanoparticles [8]. Hybrid materials usually require multi-step synthesis processes and microfluidic reactors have the ability to perform those steps separately in a controlled environment and thereby can produce homogeneous size distribution [7]. Among the studies about microfluidic synthesis of composite and hybrid nanoparticles, a number of them focused on obtaining particles with a polymer component. For instance, Zhang et al synthesized PDMS coated gold nanoparticles [9]; Hwang et al synthesized magnetic hydrogel microparticles [10]; Abou-Hassan et al utilized a multi step microreactor and coated iron oxide with silica with an average size of $50 \mathrm{~nm}$ [11]; Valencia et al obtained lipid-quantum dot hybrid nanoparticles in a continuous flow microreactor [12]; Kumar et al produced dextran coated iron oxide nanoparticles for MRI applications [13]; Yang et al used a semi-microfluidic platform to synthesize chitosan coated silver nanoparticles where droplets of silver nitrate and chitosan were generated within the microfluidic device whereas the reaction was completed outside of the reactor in a sodium hydroxide bath [14]; Köhler et al synthesized SERS active sensor polyacrylamide microparticles with silver nanoparticles [15]; Feng et al obtained lipid-polymer nanohybrids [16]; Hassan et al formed Janus particles composed of silica and gold within a droplet-based microreactor [17]; and Li et al used a flow focusing device to produce lipid-polymer hybrid nanoparticles [18]. There are also studies that focused on the synthesis of metal-metal and metal-metal 
oxide nanohybrid particles. Co@Au core shell nanohybrids [19, 20], $\mathrm{Fe} @ \mathrm{Fe}_{3} \mathrm{O}_{4}$ nanoparticles [21], PtSn alloyed nanoparticles [22], Pt-Pb core shell nanohybrids [23], Ag-ZnO nanohybdrids [24], and Ag-CoFe hybdrid nanoparticles [25] are among these synthesized particles.

This paper focuses on synthesizing hybrid nanoparticles composed of chitosan and iron oxide by a multi-step process carried in a novel microfluidic reactor. Chitosan, a biopolymer, is a well known material for its biocompatibility, low toxicity, biodegradability and stability which makes it very suitable for medical and pharmaceutical applications, especially in drug delivery [26-29]. When it is synthesized in nanometer dimensions, due to its small size and $\mathrm{pH}$ responsive chemistry it can penetrate into cells to release the drug it carries [28]. On the other hand, magnetic nanoparticles behave as a single domain material and therefore when there is an applied external magnetic field, they act like a paramagnet. Therefore, nanoparticles composed of both a magnetic material and chitosan are promising for applications such as drug delivery and magnetic resonance imaging making them both a therapeudic and a diagnostic tool [30].

Chitosan coated iron-oxide nanoparticles have been synthesized previously with batch-wise synthesis methods. Unsoy et al synthesized chitosan coated iron oxide nanoparticles and investigated the effect of the concentration of ammonia solution on the size of the particles [31]. The diameter of the synthesized nanoparticles were ranging between $2 \mathrm{~nm}$ and 8 $\mathrm{nm}$. Safari et al obtained chitosan coated iron-oxide nanoparticles using a similar approach with an average diameter of $10 \mathrm{~nm}$ and used these particles as catalyst for the synthesis of phenytoin derivatives [32]. Zarnegar et al synthesized and reported the use of composite $\mathrm{Fe}_{3} \mathrm{O}_{4} @$ chitosan nanoparticles as catalyst for the synthesis of 2, 4, 5-trisubstituted imidazoles [33]. He et al described a single step and two step process for the synthesis of chitosan decorated iron-oxide nanoparticles with an average size of $300 \mathrm{~nm}$ and reported their performance for the removal of furfural from aqueous solutions [34].

There is still a need to improve the size distribution of hybrid nanoparticles composed of chitosan and iron-oxide in literature. In order to obtain monodisperse particles with desired sizes a new microfluidic platform is introduced in this article that is capable of generating alternating droplets of reagents followed by merging and mixing them in controlled amounts. This multi-step microreactor can also be used in the synthesis of other hybrid nanomaterials that require processing of more than two reagents.

\section{Experimental}

\subsection{Materials}

Chitosan, ferric chloride hexahydrate $\left(\mathrm{FeCl}_{3} \cdot 6 \mathrm{H}_{2} \mathrm{O}\right)$ and ferrous chloride tetrahydrate $\left(\mathrm{FeCl}_{2} \cdot 4 \mathrm{H}_{2} \mathrm{O}\right)$ were obtained from Sigma Aldrich. Synthesis was performed by following a similar method described by $\mathrm{He}$ et al [34]. Solutions were prepared by using deionized (DI) water. $0.75 \mathrm{~g}$ of chitosan was mixed and stirred in $100 \mathrm{ml}$ of $0.3 \%$ acetic acid aqueous solution for 30 minutes (dispersed phase 1). $1.09 \mathrm{~g}$ of $\mathrm{FeCl}_{3} \cdot 6 \mathrm{H}_{2} \mathrm{O}$

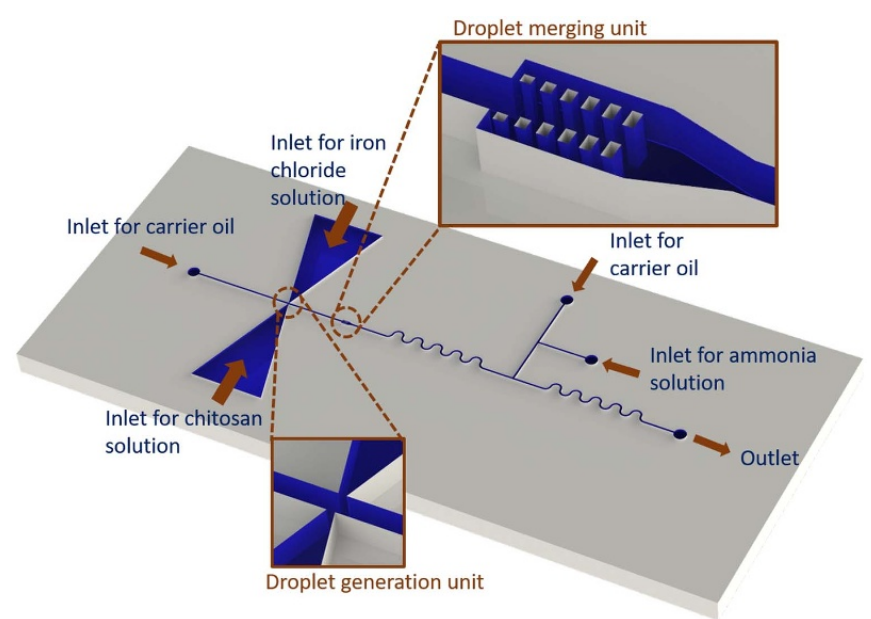

Figure 1. Schematic of the microreactor.

and $0.4 \mathrm{~g}$ of $\mathrm{FeCl}_{2} \cdot 4 \mathrm{H}_{2} \mathrm{O}$ were mixed and stirred in $100 \mathrm{ml}$ of DI water for 30 minutes (dispersed phase 2). As a third reagent solution, $10 \%$ aqueous ammonia solution is used (dispersed phase 3). Silicon oil was used as the continuous phase in the channels.

\subsection{Fabrication of the microfluidic device}

Microfluidic device was fabricated out of polydimethylsiloxane (PDMS) by using conventional soft-lithography technique. The master mold was made out of negative photoresist (SU-8 2050, Microresist Technology) on a polished silicon wafer. Microfluidic channels obtained in PDMS were bonded to a glass slide after oxygen plasma treatment.

\subsection{Experimental set-up}

Reagents were delivered to the microreactor by using syringe pumps. An inverted microscope with a camera was used to observe and record the flow.

\section{Design of the microfluidic device}

The microfluidic device is designed to carry out a multi-step synthesis by utilizing a droplet-based flow. First, alternating droplets of dispersed phase 1 and 2 are generated at the double T-junction. Then these two reagents are merged and mixed. At the final step, a droplet of dispersed phase 3 is added to the mixture to precipitate iron-oxide and complete the reaction. These steps are explained in detail below.

\subsection{Alternating droplet generation}

In the synthesis of hybrid nanoparticles of iron oxide and chitosan, three reagent solutions need to be mixed. In order to control the mixing ratio precisely and to prevent channel contamination, a droplet-based flow is utilized in channels. First, droplets of iron chloride and chitosan solutions are generated alternatively at a T-junction with tapered inlets located across 


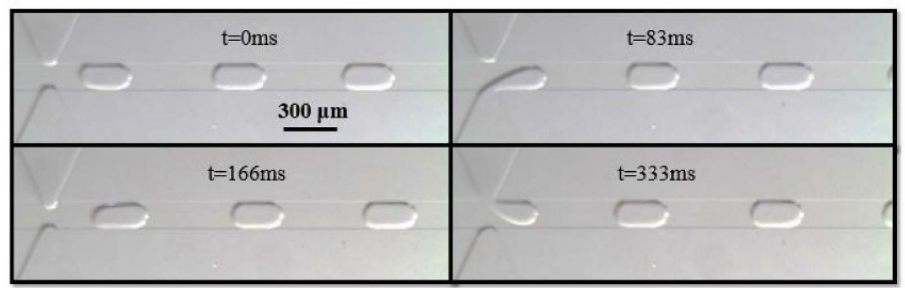

Figure 2. Alternating reagent droplet generation using tapered inlets. Tapered geometry controls the pressure at the junction which enables the formation of droplets in a sequence.

Table 1. Flow rate condition for optimum results.

\begin{tabular}{ll}
\hline Reagent & Flow rate $\left(\mu 1 \mathrm{~min}^{-1}\right)$ \\
\hline Carrier oil & 1.5 \\
Iron chloride solution & 0.17 \\
Chitosan solution & 0.12 \\
\hline
\end{tabular}

from each other. Saqib et al showed the taper angle assures an alternating droplet generation from opposing inlets due to the control of Laplace pressure and optimized the angle of taper as $25^{\circ}$ to obtain stable pattern generation and uniform droplet size and spacing [35]. As chitosan and iron chloride solution need to be mixed in 1:1 ratio, it was made sure that the pattern of alternating droplets is repetitive. As shown in figure 2 , first the lower stream (dispersed phase 2) enters the main channel, forms a droplet and afterwards the upper stream (dispersed phase 1) generates the next droplet. This process continues until flow is stopped.

In adjusting the flow rates of reagents, viscosity has to be taken in account as well. As chitosan solution has higher viscosity than iron chloride solution, it is essential to keep flow rate of iron chloride solution to be high in order to achieve droplet generation with the same volume. Table 1 summarizes the flow rates for which the best results were obtained.

These flow rates not only define the pattern but are also essential for homogeneous droplet size formation, thereby influencing the success of the reaction. It is to be noted that for alternating droplet generation, the flow ratio plays a vital role rather than the individual flow rates. But as the alternating droplet generation platform is linked with droplet merging platform, which is influenced by carrier oil flow rate, optimum values were finalized after observing the effect on both the droplet generation and merging.

\subsection{Droplet merging}

After generating the alternating droplets of two reagents, two consecutive droplets of iron chloride and chitosan solutions are merged to initiate the synthesis. An expanded channel with pillars ensures the merge of the same number of droplets in a repetitive and controlled way without disturbing the rest of the flow in the system [36]. This passive geometry prevents a third droplet to join the other two droplets which assures the same concentration in each merged droplet.

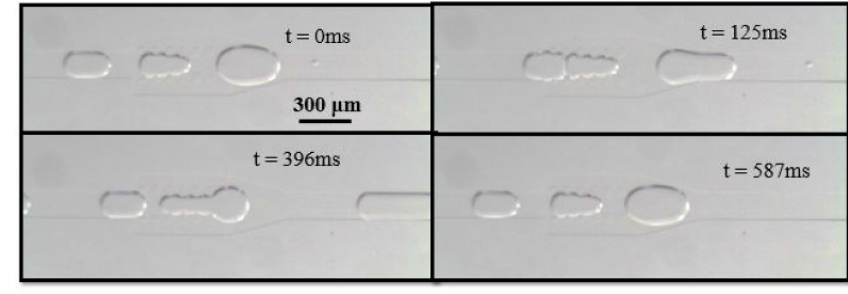

Figure 3. Passive merging of alternating droplets in pillar induced structure.

In the merging unit, the width of the pillared channel increases while the width of the pillars increases as well. Increase in the cross sectional area causes a decrease in the flow rate which also slows down the motion of droplets to an extent where they make contact and eventually merge.

Pillar induced merging geometry is optimized for merging to take place with only two consecutive droplets without any side flow. If a part of droplet flows from sides it means that the concentration of reagent is changed and the reaction parameters are no longer the same. Hence it was essential to optimize the dimensions. For this purpose the width of the channel was designed such that it increased from $150 \mu \mathrm{m}$ to $335 \mu \mathrm{m}$ and the width of the pillars were increased by $10 \mu \mathrm{m}$ for first four pillars and then by $5 \mu \mathrm{m}$ for the rest. In this case two consecutive droplets merged successfully without any side flows.

Figure 3 shows the merging of two consecutive reagent droplets. Merged droplet later passes through serpentine channels where mixing takes place due to the circulating flow profile within droplets [37]. The height of microchannel throughout the microfluidic device is $100 \mu \mathrm{m}$. In the serpentine channel the width is kept uniform at $150 \mu \mathrm{m}$ which is the same throughout the device except the merging unit. The outer radius is $450 \mu \mathrm{m}$ and the inner radius is $300 \mu \mathrm{m}$, resulting with a mean radius of $375 \mu \mathrm{m}$. There are 11 arcs after the merging geometry with a length of approximately $1.3 \mathrm{~cm}$ and 12 arcs after the addition of ammonia with length of approximately $1.4 \mathrm{~cm}$

\subsection{Addition of the third reagent}

In the final step of the synthesis, ammonia solution is added as a third reagent to precipitate iron oxide. First, a droplet of ammonia solution is generated at a T-junction and later it is fused with the droplet composed of chitosan and iron chloride 


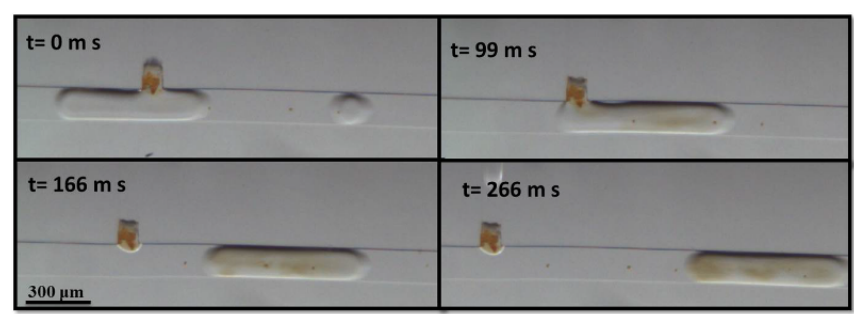

Figure 4. Merging of ammonia droplet to initiate reaction.
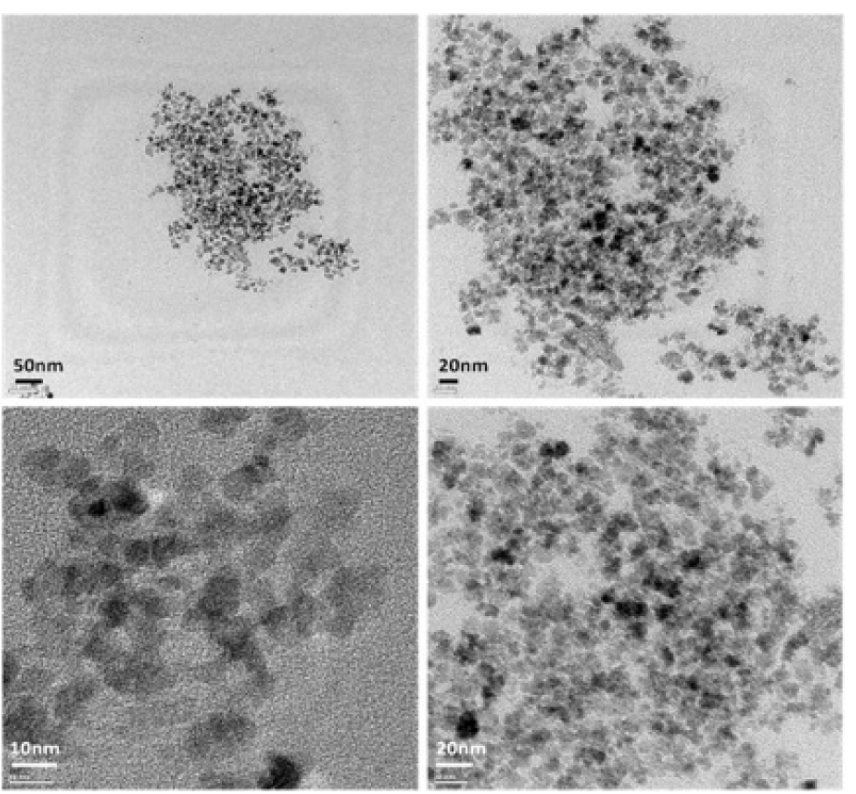

Figure 5. TEM images of synthesized nanoparticles.

solution. As soon as the ammonia solution is added, oxidation of iron is observed with the color change within droplets as shown in figure 4.

The amount of added ammonia solution is essential in the size of the synthesized nanoparticles. Unsoy et al showed that increasing concentration of ammonia solution decreases the size of nanoparticles [31].

\section{Results and discussion}

\subsection{Results}

To understand their both physical characteristics and chemical composition, nanoparticles synthesized in the microreactor were characterized by various analytical tools including transmission electron microscopy (TEM), energy dispersive $\mathrm{X}$-ray analysis (EDX) and Fourier transform infrared spectroscopy (FTIR).

Images of the particles were recorded with TEM and average size of nanoparticles were measured as $8.5 \mathrm{~nm}$ with a standard deviation of $1.3 \mathrm{~nm}$ after image processing of the data. TEM images are shown in figure 5. The size of these particles are smaller than the ones synthesized by batch techniques reported in literature [31,34] mainly due to having a
Table 2. EDX data of synthesized nanoparticles.

\begin{tabular}{ll}
\hline Element & Percentage \\
\hline $\mathrm{Fe}$ & 44.19 \\
0 & 55.8 \\
\hline
\end{tabular}



Figure 6. FTIR spectra of synthesized nanoparticles.

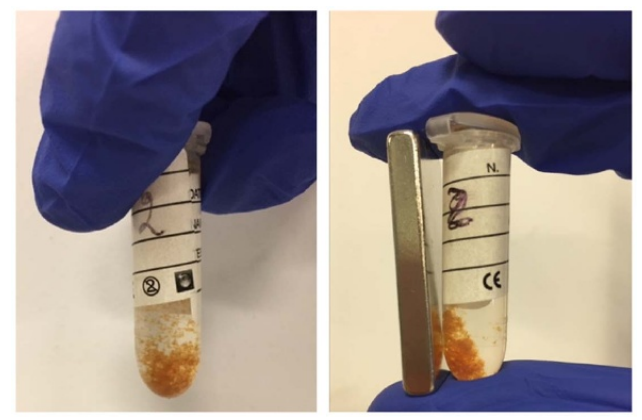

Figure 7. Nanoparticles without and with the presence of a magnet.

synthesis constrained in droplets as small as $19 \mathrm{~nL}$. On the other hand, an improved size distribution is one of the major advantages of using microfluidic systems which is a result of maintaining a uniform concentration and residence time for all reagents. These images also show that particles are spherical; on the other hand, since ligands were not used during the synthesis there is agglomeration.

EDX analysis was performed to confirm the existence of iron-oxide component in the nanoparticles. Only the elements $\mathrm{Fe}$ and $\mathrm{O}$ were selected to be scanned in the system. Since the grid structure used for holding the sample was made out of carbon, scanning of carbon was excluded from the analysis as it would not result in an accurate conclusion for chitosan. EDX data is presented in table 2 and shows the percentage distribution of $\mathrm{Fe}$ to $\mathrm{O}$. In order to confirm the presence of chitosan, FTIR analysis was made and the result is shown in figure 6. Successful synthesis of hybrid chitosan and ironoxide nanoparticles is confirmed by the appearance of characteristic bands at $3328.94 \mathrm{~cm}^{-1}$ indicating the bonding of N-H group with $\mathrm{O}-\mathrm{H}$ group in chitosan and $1629.45 \mathrm{~cm}^{-1}$ indicating the $\mathrm{C}=\mathrm{O}$ of alcoholic group in chitosan.

The synthesized nanoparticles were also magnetic as shown in figure 7 . 


\subsection{Discussion}

The analytical data obtained from TEM, FTIR and EDX confirm the successful synthesis of nanoparticles composed of chitosan and iron oxide. Compared to the batch synthesized chitosan coated iron-oxide nanoparticles reported by He et al [34], the nanoparticles produced in the microreactor were two orders of magnitude smaller in size. A size distribution data was not reported in [34], therefore a direct comparison is not possible to be made; on the other hand, similar particle agglomeration was observed as the synthesis was carried out without any surfactant or ligand in both cases. Compared to another batch method reported for the synthesis of chitosan coated iron-oxide nanoparticles by Unsoy et al [31], it is observed that size distribution was improved with the microreactor. Unsoy et al also observed agglomeration of nanoparticles.

\section{Conclusion}

Synthesis of hybrid chitosan with iron-oxide nanoparticles has been realized by using a microfluidic platform which resulted in a narrower size distribution and reduced residence time compared to the batch-wise methods. Alternating droplet formation, controlled merging and step by step mixing was carried out in a single microfluidic chip. Dimensions of the device were optimized and flow rate of reagents were adjusted to meet the requirements of the synthesis recipe. The designed microfluidic device can be used to produce other materials that require a multi-step synthesis protocol.

\section{Conflicts of interest}

Authors declare no conflict of interest.

\section{Acknowledgments}

This work was funded by the Science Academy Young Scientists Award (BAGEP 2016). We also thank Eliza Sopubekova for her assistance in FTIR and Mr Mustafa Guler for his assistance in TEM analysis.

\section{ORCID iD}

E Yegan Erdem (DD https://orcid.org/0000-0001-9852-2293

\section{References}

[1] Pan Y, Xue P, Liu S, Zhang L, Guan Q and Zhu J et al 2018 Metal-based hybrid nanoparticles as radiosensitizers in cancer therapy Colloid. Interface Sci. Commun. 23 45-51

[2] Waiskopf N, Ben-Shahar Y and Banin U 2018 Photocatalytic hybrid semiconductor-metal nanoparticles; from synergistic properties to emerging applications Adv. Mat. 301706697

[3] Jouyandeh M, Jazani O M, Navarchian A H, Shabanian M, Vahabi H and Saeb M R 2019 Bushy-surface hybrid nanoparticles for developing epoxy superadhesives Appl. Surf. Sci. 479 1148-60
[4] Chang C H, Paul B K, Remcho V T, Atre S and Hutchison J E 2008 Synthesis and post-processing of nanomaterials using microreaction technology J Nanopart. Res. 10 965-80

[5] Zhao C X, He L, Qiao S Z and Middelberg A P 2011 Nanoparticle synthesis in microreactors Chem. Eng. Sci. 66 1463-79

[6] Erdem E Y, Cheng J C, Doyle F M and Pisano A P 2014 Multi-temperature zone, droplet-based microreactor for increased temperature control in nanoparticle synthesis Small 10 1076-80

[7] Hung L H and Lee A P 2007 Microfluidic devices for the synthesis of nanoparticles and biomaterials J. Med. Biol. Eng. 271

[8] Wang J and Song Y 2017 Microfluidic Synthesis of Nanohybrids Small 131604084

[9] Zhang Q, Xu J J, Liu Y and Chen H Y 2008 In-situ synthesis of poly (dimethylsiloxane)-gold nanoparticles composite films and its application in microfluidic systems Lab Chip. $8352-7$

[10] Hwang D K, Dendukuri D and Doyle P S 2008 Microfluidic-based synthesis of non-spherical magnetic hydrogel microparticles Lab Chip. 8 1640-7

[11] Abou-Hassan A, Bazzi R and Cabuil V 2009 Multistep continuous-flow microsynthesis of magnetic and fluorescent -Fe2O3@ SiO2 core/shell nanoparticles Angew. Chem. Int. Ed. 48 7180-3

[12] Valencia P M, Basto P A, Zhang L, Rhee M, Langer R and Farokhzad O C et al 2010 Single-step assembly of homogenous lipid-polymeric and lipid-quantum dot nanoparticles enabled by microfluidic rapid mixing $A C S$ Nano 4 1671-9

[13] Kumar K, Nightingale A M, Krishnadasan S H, Kamaly N, Wylenzinska-Arridge M and Zeissler K et al 2012 Direct synthesis of dextran-coated superparamagnetic iron oxide nanoparticles in a capillary-based droplet reactor J. Mater. Chem. 22 4704-8

[14] Yang C H, Wang L S, Chen S Y, Huang M C, Li Y H and Lin Y C et al 2016 Microfluidic assisted synthesis of silver nanoparticle-chitosan composite microparticles for antibacterial applications Int. J. Pharm. 510 493-500

[15] Köhler J M, März A, Popp J, Knauer A, Kraus I and Faerber J et al 2013 Polyacrylamid/silver composite particles produced via microfluidic photopolymerization for single particle-based SERS microsensorics Anal. Chem. 85 313-18

[16] Feng Q, Zhang L, Liu C, Li X, Hu G and Sun J et al 2015 Microfluidic based high throughput synthesis of lipid-polymer hybrid nanoparticles with tunable diameters Biomicrofluidics 9052604

[17] Hassan N, Stocco A and Abou-Hassan A 2015 Droplet liquid/liquid interfaces generated in a microfluidic device for assembling Janus inorganic nanohybrids J. Phys. Chem. C 119 10758-65

[18] Li Y, Huang X, Lee R J, Qi Y, Wang K and Hao F et al 2016 Synthesis of polymer-lipid nanoparticles by microfluidic focusing for siRNA delivery Molecules 21 1314-22

[19] Song Y, Wang Y, Ji S and Ding J 2012 Shell-driven fine structure transition of core materials in Co@Au core-shell nanoparticles Nano-Micro Lett. 4 235-42

[20] Song Y, Ding J and Wang Y 2012 Shell-dependent evolution of optical and magnetic properties of Co@Au core-shell nanoparticles J. Phys. Chem. C 116 11343-50

[21] Song Y, Ji S, Song Y J, Li R, Ding J and Shen X et al 2013 In situ redox microfluidic synthesis of core-shell nanoparticles and their long-term stability J. Phys. Chem. C 117 17274-84

[22] Wu F, Zhang D, Peng M, Yu Z, Wang X and Guo G et al 2016 Microfluidic synthesis enables dense and uniform loading of surfactant-free PtSn nanocrystals on carbon supports for 
enhanced ethanol oxidation Angew. Chem. Int. Ed. 55 4952-6

[23] Sebastián V and Jensen K F 2016 Nanoengineering a library of metallic nanostructures using a single microfluidic reactor Nanoscale 8 15288-95

[24] Taoab S, Yang M, Chenab H, Renab R and Chen G 2016 Continuous synthesis of hedgehog-like $\mathrm{Ag}-\mathrm{ZnO}$ nanoparticles in a two-stage microfluidic system RSC Adv. $645503-11$

[25] Ma J, Wang J, Zhang G, Peng L, Song X and Ding J et al 2019 Magnetic and optical properties of $\mathrm{Ag}-\mathrm{CoFe}$ nanohybrids prepared by a sequenced microfluidic process ChemistrySelect 4 14157-61

[26] Lee K H, Shin S J, Kim C B, Kim J K, Cho Y W and Chung B G et al 2010 Microfluidic synthesis of pure chitosan microfibers for bio-artificial liver chip Lab Chip. 10 1328-34

[27] Muzzarelli R A 2011 Biomedical exploitation of chitin and chitosan via mechano-chemical disassembly, electrospinning, dissolution in imidazolium ionic liquids and supercritical drying Mar. Drugs 9 1510-33

[28] Nagpal K, Singh S K and Mishra D N 2010 Chitosan nanoparticles: a promising system in novel drug delivery Chem. Pharm. Bull. 58 1423-30

[29] Couvreur P 2013 Nanoparticles in drug delivery: past, present and future Adv Drug Delivery Rev. 65 21-3
[30] Sailor M and Park J H 2012 Hybrid nanoparticles for detection and treatment of cancer Adv Mater. 24 3779-802

[31] Unsoy G, Yalcin S, Khodadust R, Gunduz G and Gunduz U 2012 Synthesis optimization and characterization of chitosan-coated iron oxide nanoparticles produced for biomedical applications J. Nanopart Res. 14964

[32] Safari J and Javadian L 2014 Chitosan decorated Fe 3 O 4 nanoparticles as a magnetic catalyst in the synthesis of phenytoin derivatives $R S C A d v .4$ 48973-9

[33] Zarnegar Z and Safari J 2014 Fe 3 O 4@ chitosan nanoparticles: a valuable heterogeneous nanocatalyst for the synthesis of 2, 4, 5-trisubstituted imidazoles RSC Adv. $420932-9$

[34] He Y, Pei M, Du Y, Yu F, Wang L and Guo W 2014 Synthesis, characterization and application of chitosan coated $\mathrm{Fe}_{3} \mathrm{O}_{4}$ particles as an adsorbent for the removal of furfural from aqueous solution $R S C A d v .430352-7$

[35] Saqib M, Şahinoğlu O B and Erdem E Y 2018 Alternating droplet formation by using tapered channel geometry $\mathrm{Sci}$ Rep. 81606

[36] Niu X, Gulati S, Edel J B and de Mello A J 2008 Pillar-induced droplet merging in microfluidic circuits $L a b$ Chip 8 1837-41

[37] Özkan A and Erdem E Y 2015 Numerical analysis of mixing performance in sinusoidal microchannels based on particle motion in droplets Microfluid Nanofluid 19 1101-8 